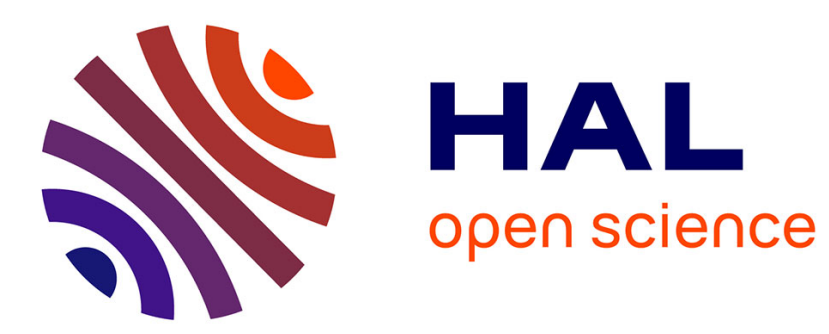

\title{
Robust nonlinear adaptive control of multiphase synchronous buck power converters
}

\author{
H. El Fadil, Fouad Giri
}

\section{To cite this version:}

H. El Fadil, Fouad Giri. Robust nonlinear adaptive control of multiphase synchronous buck power converters. Control Engineering Practice, 2009, 17, pp.1245-1254. 10.1016/j.conengprac.2009.04.009 . hal-01058704

\section{HAL Id: hal-01058704 https://hal.science/hal-01058704}

Submitted on 27 Aug 2014

HAL is a multi-disciplinary open access archive for the deposit and dissemination of scientific research documents, whether they are published or not. The documents may come from teaching and research institutions in France or abroad, or from public or private research centers.
L'archive ouverte pluridisciplinaire HAL, est destinée au dépôt et à la diffusion de documents scientifiques de niveau recherche, publiés ou non, émanant des établissements d'enseignement et de recherche français ou étrangers, des laboratoires publics ou privés. 


\title{
Robust nonlinear adaptive control of multiphase synchronous buck power converters
}

\author{
H. El Fadil *, F. Giri \\ GREYC Lab, University of Caen, Caen, France
}

Keywords:

Interleaved converters

Voltage regulation

Load sharing

Nonlinear control

Adaptive control

Robust parameter update

\begin{abstract}
A B S T R A C T
The problem of controlling multiphase synchronous buck power converters is considered. The aims are to regulate the output voltage of the converter and to ensure adequate current sharing between its different channels. Using the backstepping technique, an adaptive controller is designed based on a large-signal bilinear model of the whole multi-channel converter. A parameter projection is used to ensure that the obtained adaptive controller is robust to parasitic resistances. The controller is formally shown to meet the objectives of closed-loop asymptotic stability, output reference tracking, and equal load sharing. The attraction region depends on the uncertain parasitic impedance size, with the attraction region being larger for smaller parasitic impedances. In the ideal case of no parasitic impedances, the closed-loop asymptotic stability is global. These theoretical results are confirmed by simulation.
\end{abstract}

\section{Introduction}

Rapid evolution in microprocessor technology is creating new power supply requirements. This evolution began when the highperformance Pentium processor was invented. This processor is driven by a non-standard (less than $5 \mathrm{~V}$ ) power supply, rather than drawing its power from the $5 \mathrm{~V}$ supply on the motherboard. In order to meet the demands for faster and more efficient data processing, modern microprocessors are being designed with lower voltage requirements. The voltage supplies of future processors are expected to be below $1 \mathrm{~V}$. More devices will be packed onto a single processor chip and operated at higher frequencies (above $3 \mathrm{GHz}$ ). Therefore, microprocessors need aggressive power management, and future generations are expected to draw currents of up to $100 \mathrm{~A}$. These requirements will necessitate special power supplies and voltage regulator modules (VRMs) to provide lower voltages with higher currents and faster transient capabilities. As processors get faster, the VRMs must also be speedier. Future microprocessors are typically expected to exhibit higher current slew rates ( $5 \mathrm{~A} / \mathrm{ns})$. Such small slew rates may significantly disturb the VRM voltage in the presence of equivalent series resistance (ESR) and equivalent series inductance (ESL). When these parasitic impedances are not sufficiently small, the supplied voltage may fall out of the admissible range, especially during transient periods

\footnotetext{
* Corresponding author.

E-mail addresses: elfadilhassan@yahoo.fr (H. El Fadil), giri@greyc.ensicaen.fr (F. Giri).
}

(Zhang, Jovanovic, \& Lee, 1996). On the other hand, the imposed voltage tolerance is only $2 \%$, which means that for $1.1 \mathrm{~V}$ at VRM output, the voltage deviation must not exceed $\pm 33 \mathrm{mV}$. This requirement is a challenging problem for VRM designers.

Parallel connection of switching converters is an interesting technique from both practical and fundamental viewpoints (Chang \& Knights, 1995; Perreault \& Kassakian, 1997). Several parallel converters share the same output, contributing to the production of a large global current with a low supply voltage. This is suitable for next-generation microprocessors (Zhou, Xu, \& Lee, 2000; Panov \& Jovanovic, 2001). Load sharing is effective in improving reliability and fault tolerance; this method also ensures reduced ripple in the output current. This is convenient because it allows for reductions of the involved filter size and the losses therein. Finally, current sharing reduces switching and conduction losses, as well as electromagnetic interference (EMI).

Interleaved buck converters (IBCs) are widely used in the personal computer industry. In particular, central processing units (CPUs) are powered using VRMs. This topology is widely used, as interleaving the converters results in reduced input and output capacitor ripple currents compared to a single buck power stage. The reduction in input and output capacitor root mean square (RMS) currents makes it possible to reduce the size of the needed input and output capacitance. Parallel mode operation of dc-dc converters has been realized with multiple control schemes. In (Zhou et al., 2000), a linear VRM controller was proposed, and was shown to ensure equal load sharing between branches. However, the parasitic resistances in the output capacitors were neglected and the effects of uncertainty or changes in the converter load were totally ignored. In Huang, Schuellein, and Clavette (2003), 
experimental results were reported for a parallel buck converter controlled with feed-forward voltage mode control; however, no experimental evaluation or theoretical analysis was performed for the control performances in the presence of a changing load. In Abu-Qahouq, Mao, and Batarseh (2004), a hysteresis-type control technique was proposed for parallel buck converters. The technique is simple, but asymptotic perfect voltage regulation cannot be achieved, as the hysteresis feature causes output ripples. Furthermore, the parasitic elements in switches, inductances, and capacitances were not accounted for in this study. The study also did not include any formal analysis of closed-loop stability and performance, particularly in the presence of load variations. In Berbel, Guerrero, Cruz, Miret, and Castilla (2005), a linear regulator was designed using a linear model that neglected the R-ON resistance of switches. There was no formal analysis of the robustness of regulator stability in the presence of a changing load. The regulation performances were only illustrated through simulations. In Saito, Tasaki, and Torikai, (2005), a winner-take-all switching technique was used to control the converter switches, and a chaos analysis was presented. It was shown that current ripples can be reduced by making a judicious choice of the switching rule. However, the study contained no formal proof that equal load sharing is guaranteed, or that robustness with respect to load change is ensured.

The present paper focuses on the problem of controlling interleaved, synchronous, PWM buck converters. The controller is obtained directly from the large-signal bilinear model of the whole system, which involves some uncertain parameters. An adaptive controller is designed with the backstepping technique; the goals of the controller are closed-loop stability, tight output voltage regulation, fast transient response, and equal current sharing between modules. Parameter adaptation is limited to the load resistance, which is the only parameter that varies. The remaining uncertain parameters correspond to parasitic impedances, which in practice are quite small. The effects of these parasitic elements are considered as modeling errors. These errors may cause parameter drift if standard update laws are used. Therefore, a modified parameter adaptive law involving a projection onto a convex set containing the true value of the unknown parameter is chosen. The adaptive controller thus obtained is robust, and its performance can be formally shown to achieve the design specifications. More specifically, the closedloop system is given a state space representation; the state variables are the tracking errors of output voltage and current and parameter estimation error. It is proven that this closed-loop system is asymptotically stable, with an attraction region that depends on the parasitic impedances; the smaller these impedances, the larger the attraction region. In the ideal situation of no parasitic impedances, the attraction region extends to the whole state space, meaning that the system exhibits global asymptotic stability. These theoretical results are confirmed by numerical simulations on the Texas Instruments Evaluation Module TPS40090EVM-002.

The paper is organized as follows: in Section 2, the interleaved synchronous buck converter is described and modeled. Controller design and analysis are dealt with in Sections 3 and 4, respectively. In Section 5, controller tracking performances are illustrated through numerical simulations. A conclusion and list of references end the paper.

\section{Multiphase synchronous buck converter modeling}

Fig. 1 shows the topology of a multiphase synchronous buck converter. It consists of $N$ synchronous buck converters connected in parallel, all sharing the common load represented by the pure

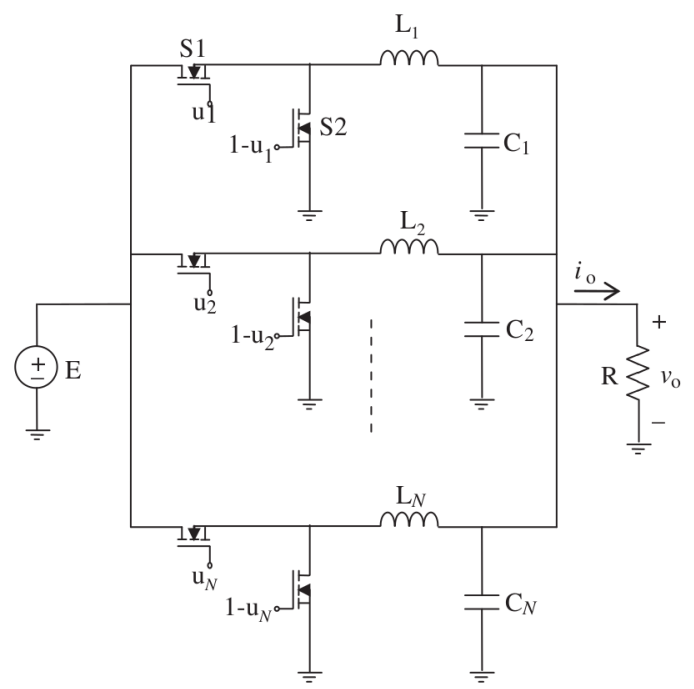

Fig. 1. Multi-phase interleaved buck converter.

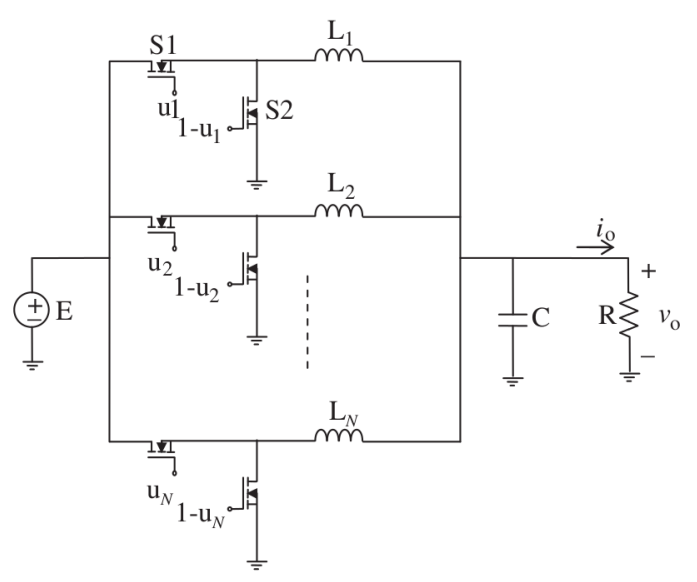

Fig. 2. Equivalent circuit of interleaved buck converter.

resistor $R$, which corresponds to the microprocessor load. The $k$ th converter $(k=1, \ldots, N)$ includes synchronous switches, an inductance $L_{k}$, and a capacitor $C_{k}$. Due to their lower voltage drop, synchronous switches are much more efficient than conventional Schottky diodes in applications necessitating high current at low voltage. Each converter is controlled using an interleaved PWM. A phase shift of $(360 / N)$ degrees is introduced between each channel. The overall current of the converter is then the addition of $N$ pulsating currents, each with a $(360 / N)$ phase. The ripple frequency of the total current is $N$ times the fundamental switching frequency of a single converter. Therefore, the total current ripple turns out to be smaller than the current ripple of an individual converter. For a given current ripple, interleaving the channels allows for much smaller and lighter inductances. As the capacitors $C_{k}(k=1, \ldots, k)$ are all in parallel, the scheme of Fig. 1 can be redrawn as shown in Fig. 2, with $C=\sum_{k=1}^{N} C_{k}$.

Fig. 3 shows an averaged equivalent model of the $k$ th single synchronous buck converter, where $R_{1}$ and $R_{2}$ represent the R-ON of switches S1 and S2, respectively; $r_{L k}$ and $r_{C k}$ are the ESR values of $L_{k}$ and $C_{k}$, respectively. Fig. 2 also defines the control input $u$ of the synchronous buck converter. This variable takes the discrete value $u_{k}=1$ when switch S1 is on and S2 is off, and $u_{k}=0$ when switch S1 is off and S2 is on. For simplicity, the ESL of $C_{k}$ is not taken into account, as it only affects the high frequency spike, as 


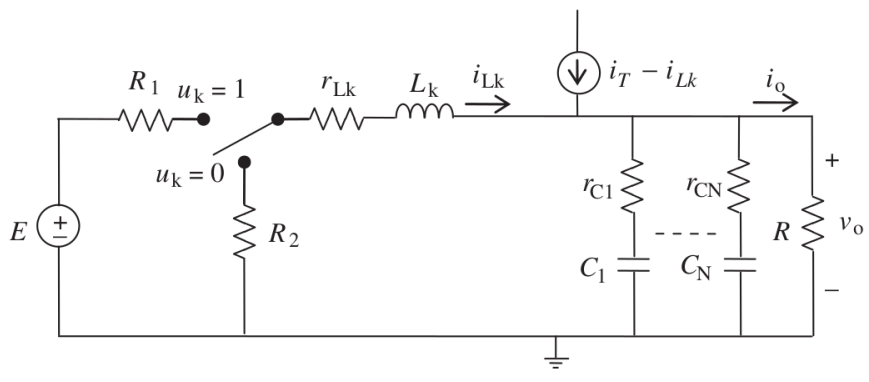

Fig. 3. Average equivalent circuit of a single buck converter.

shown in Berbel et al. (2005). The current source $\left(i_{T}-i_{L k}\right)$ represents the sum of all supplied currents from the other cells. Considering identical inductances and capacitors, the following expressions can be obtained:

$r_{L 1}=r_{L 2}=\cdots=r_{L N}=r_{L}$

$r_{C 1}=r_{C 2}=\cdots=r_{C N}=r_{C}$

$L_{1}=L_{2}=\cdots=L_{N}=L$

$C_{1}=C_{2}=\cdots=C_{N}=C$

Applying Kirchoff's laws to the circuit of Fig. 3, and using the averaging technique (Iannelli, Johansson, Jönsson, \& Vasca, 2008; Krein, Bentsman, Bass, \& Lesieutre, 1990) yield the following average model of the $k$ th converter:

$\frac{d \bar{i}_{L k}}{d t}=-\frac{1}{L}\left[R_{L}+R_{2}+\left(R_{1}-R_{2}\right) \mu_{k}\right] \bar{i}_{L k}-\frac{1}{L} \bar{v}_{0}+\frac{E}{L} \mu_{k}$

$\frac{d \bar{v}_{0}}{d t}=\frac{1}{C_{e}} \bar{i}_{T}-\frac{1}{R C_{e}} \bar{v}_{0}+\zeta(t)$

With

$$
\begin{aligned}
\zeta(t)= & -\frac{R_{C}}{\left(R+R_{C}\right) C_{e}}\left(\bar{i}_{T}-\frac{\bar{v}_{0}}{R}\right)+\left(R_{C} / / R\right) \frac{d \bar{i}_{T}}{d t} \\
= & R_{C}\left\{-\frac{1}{\left(R+R_{C}\right)}\left[\frac{R\left(R_{L}+R_{2}\right)}{L}+\frac{1}{C_{e}}\right] \bar{i}_{T}-\frac{1}{\left(R+R_{C}\right)}\left[\frac{N R}{L}-\frac{1}{R C_{e}}\right] \bar{v}_{0}\right. \\
& \left.+\frac{R}{\left(R+R_{C}\right) L}\left[-\left(R_{1}-R_{2}\right) \sum_{k=1}^{N} \bar{i}_{L k} \mu_{k}+E \sum_{k=1}^{N} \mu_{k}\right]\right\}
\end{aligned}
$$

and

$\bar{i}_{T}=\sum_{k=1}^{N} \bar{i}_{L k}$

where $N$ denotes the number of units connected in parallel, $\bar{i}_{L k}$ is the average value of the current in the inductor $L_{k}, \bar{v}_{0}$ the average value of the output voltage, and $\mu_{k}$ the average value of the discrete control $u_{k}$, with values in the range [0 1 ]. The continuous signal $\mu_{k}$, usually called the duty ratio, acts as the input control variable of the $k$ th module. The parameters $R_{L}, R_{C}$, and $C_{e}$ are defined as follows:

$R_{L}=r_{L} ; \quad R_{C}=\frac{r_{C}}{N} ; \quad C_{e}=\sum_{k=1}^{N} C_{k}=N C$

The function $\zeta(t)$ represents an output disturbance in (2a) and (2b). In (2c), it is seen that $\zeta(t)$ arises as a consequence of parasitic resistances in the capacitors. Furthermore, $\zeta(t)$ converges to zero in steady state (after the output voltage has converged). The adaptive regulator under development must be robust to the presence of this disturbance.

\section{Adaptive controller design}

In this study, the load resistance $R$ in model (2) may be subject to infrequent jumps. This phenomenon is especially relevant in microprocessor power supply devices. The load jumps occur when microprocessors switch from sleep mode to active mode, and vice versa. To cope with this uncertain parameter, the controller will be given some capacity to learn. More specifically, the designed adaptive controller must involve on-line estimation of the unknown parameter

$\theta=\frac{1}{R}$

The corresponding estimate is denoted $\hat{\theta}$, and the estimation error is

$\tilde{\theta}=\theta-\hat{\theta}$

The control scheme seeks to fulfill the following three objectives: asymptotic stability of the closed loop system, tight regulation of the voltage $v_{0}$, and fast transient response and proper current sharing. To this end, an adaptive nonlinear regulator will be designed using the backstepping approach. Closely following (Krstić, Kanellakopoulos, \& Kokotović, 1995), the controller is designed in two steps.

Step 1 . The following tracking error is introduced:

$z_{1}=\bar{v}_{0}-V_{d} \quad\left(V_{d} \neq 0\right)$

where $V_{d}$ denotes the constant desired output reference value. ${ }^{1}$ Achieving the tracking objective means forcing the error $z_{1}$ to vanish. To this end, the dynamics of $z_{1}$ need to be clearly defined. Differentiating (6) with respect to time, one obtains from (2b):

$\dot{z}_{1}=\frac{1}{C_{e}} \bar{i}_{T}-\frac{\theta}{C_{e}} \bar{v}_{0}+\zeta$

In the above equation, the quantity $\bar{i}_{T} / C_{e}$ stands as a virtual control variable. Consider the following Lyapunov function:

$V_{1}=0.5 z_{1}^{2}+0.5 \tilde{\theta}^{2} / \gamma$

where $\gamma>0$ is any real constant, called the parameter adaptation gain. The time-derivative of $V_{1}$ along the trajectory of (7) is

$\dot{V}_{1}=z_{1}\left(\frac{1}{C_{e}} \bar{i}_{T}+w_{1} \hat{\theta}\right)-\frac{\tilde{\theta}}{\gamma}\left(\dot{\hat{\theta}}-\gamma w_{1} z_{1}\right)$

where the first regressor function is defined by

$w_{1}=-\bar{v}_{0} / C_{e}$

One can eliminate $\tilde{\theta}$ from $\dot{V}_{1}$ with the update law $\dot{\hat{\theta}}=\gamma \tau_{1}$, where

$\tau_{1}=w_{1} z_{1}$

Furthermore, $z_{1}$ can be controlled to zero if $\bar{i}_{T} / C_{e}=\alpha_{1}$, where $\alpha_{1}$ is a stabilizing function defined by

$\alpha_{1}=-w_{1} \hat{\theta}-c_{1} z_{1}$

where $c_{1}>0$ is a design parameter. Since $\bar{i}_{T} / C_{e}$ is not the actual control input, one can only seek convergence of the error $\bar{i}_{T} / C_{e}-$ $\alpha_{1}$ to zero, and $\dot{\hat{\theta}}=\gamma \tau_{1}$ is not taken as a parameter update law. Nevertheless, $\tau_{1}$ is retained as the first tuning function, and the presence of $\tilde{\theta}$ in $\dot{V}_{1}$ is tolerated. Keeping the current sharing requirement in mind, the following second error variables

\footnotetext{
${ }^{1}$ For the sake of simplicity, only constant references are considered. Nevertheless, subsequent controller design can easily be extended to general reference signals provided these are twice derivable with respect to time and their two first derivatives are known, bounded and piecewise continuous.
} 
are defined:

$z_{2 k}=\bar{i}_{L k} / C_{e}-\alpha_{1} / N, \quad k=1, \ldots, N$

The next step is to determine a control law for each control signal $\mu_{k}$ and an adaptive law for the parameter estimate $\hat{\theta}$, so that the set of errors $\left\{z_{1}, z_{2 k} ; k=1, \ldots, N\right\}$ vanish asymptotically. First, the derivatives $\dot{z}_{1}$ and $\dot{V}_{1}$ must be expressed as functions of the new errors $z_{2 k},(k=1, \ldots, N)$. Adding both sides of all equalities in (13), and using (2d), yields

$\sum_{j=1}^{N} z_{2 k}=\bar{i}_{T} / C_{e}-\alpha_{1}$

Then, using (12) and (14), Eq. (7) becomes

$\dot{z}_{1}=-c_{1} z_{1}+\sum_{k=1}^{N} z_{2 k}+w_{1} \tilde{\theta}+\zeta$

The derivative (9) of the Lyapunov function is also rewritten:

$\dot{V}_{1}=-c_{1} z_{1}^{2}+z_{1} \sum_{k=1}^{N} z_{2 k}+\tilde{\theta}\left(\tau_{1}-\dot{\hat{\theta}} / \gamma\right)+z_{1} \zeta$

Step 2. The objective is now to make the error variables $\left\{z_{1}, z_{2 k}(k=1, \ldots, N)\right\}$ vanish asymptotically. To this end, the dynamics of $z_{2 k}$ are first determined. Differentiating (13) and using (2a), (12), and (15), the following equation is obtained:

$$
\begin{aligned}
\dot{z}_{2 k}= & \frac{1}{L C_{e}}\left[E-\left(R_{1}-R_{2}\right) \bar{i}_{L k}\right] \mu_{k}+w_{2} \tilde{\theta}-\frac{1}{L C_{e}}\left(R_{L}+R_{2}\right) \bar{i}_{L k} \\
& -\left(\frac{1}{L C_{e}}-\frac{\hat{\theta}^{2}}{N C_{e}^{2}}\right) \bar{v}_{0}-\frac{\hat{\theta}}{N C_{e}^{2}} \bar{i}_{T} \\
& +\frac{w_{1}}{N} \dot{\hat{\theta}}-\frac{C_{1}^{2}}{N} z_{1}+\frac{c_{1}}{N} \sum_{k=1}^{N} z_{2 k}+\frac{1}{N}\left(c_{1}-\frac{\hat{\theta}}{C_{e}}\right) \zeta
\end{aligned}
$$

where $w_{2}$ represents the second regressor function, defined as follows:

$w_{2}=\frac{\left(c_{1}-\hat{\theta} / C_{e}\right) w_{1}}{N}$

In (17), the actual control inputs $\mu_{k}$ arise explicitly for the first time. Now, the goal is to find a control law for the $\mu_{k}$ 's and an adaptive law for $\hat{\theta}$, so that the $\left(z_{1}, z_{21}, \ldots, z_{2 N}, \tilde{\theta}\right)$-system becomes globally asymptotically stable. To this end, consider the augmented Lyapunov function:

$V=V_{1}+\frac{1}{2} \sum_{j=1}^{N} z_{2 j}^{2}=\frac{1}{2} z_{1}^{2}+\frac{1}{2 \gamma} \tilde{\theta}^{2}+\frac{1}{2} \sum_{k=1}^{N} z_{2 k}^{2}$

Using (15)-(17), the time derivative of $V$ is

$$
\begin{aligned}
\dot{V}= & \dot{V}_{1}+\sum_{k=1}^{N} z_{2 k} \dot{z}_{2 k} \\
= & -c_{1} z_{1}^{2}+\sum_{k=1}^{N} z_{2 k}\left[z_{1}+\frac{1}{L C_{e}}\left[E-\left(R_{1}-R_{2}\right) \bar{i}_{L k}\right] \mu_{k}-\frac{1}{L C_{e}}\left(R_{L}+R_{2}\right) \bar{i}_{L k}\right. \\
& \left.-\left(\frac{1}{L C_{e}}-\frac{\hat{\theta}^{2}}{N C_{e}^{2}}\right) \bar{\nu}_{0}-\frac{\hat{\theta}}{N C_{e}^{2}} \bar{i}_{T}+\frac{w_{1}}{N} \dot{\hat{\theta}}-\frac{c_{1}^{2}}{N} z_{1}+\frac{c_{1}}{N} \sum_{k=1}^{N} z_{2 k}\right] \\
& +\tilde{\theta}\left[\tau_{1}+w_{2} \sum_{k=1}^{N} z_{2 k}-\frac{\dot{\hat{\theta}}}{\gamma}\right]+\left(z_{1}+\frac{1}{N}\left(c_{1}-\frac{\hat{\theta}}{C_{e}}\right) \sum_{k=1}^{N} z_{2 k}\right) \zeta \quad(20)
\end{aligned}
$$

The control signals $\mu_{k}$ should be chosen so that $\dot{V}$ is a negative definite function of the state variables $\left(z_{1}, z_{21}, \ldots, z_{2 N}, \tilde{\theta}\right)$. If the disturbing term $\zeta$ were null, the above expression for $\dot{V}$ would suggest the following control law:

$$
\begin{aligned}
\mu_{k}= & \frac{L C_{e}}{E-\left(R_{1}-R_{2}\right) \bar{i}_{L k}}\left\{\frac{1}{L C_{e}}\left(R_{L}+R_{2}\right) \bar{i}_{L k}+\left(\frac{1}{L C_{e}}-\frac{\hat{\theta}^{2}}{N C_{e}^{2}}\right) \bar{v}_{0}\right. \\
& \left.+\frac{\hat{\theta}}{N C_{e}^{2}} \bar{i}_{T}-\frac{w_{1}}{N} \dot{\hat{\theta}}+\left(\frac{c_{1}^{2}}{N}-1\right) z_{1}-\frac{c_{1}}{N} \sum_{k=1}^{N} z_{2 k}-c_{2} z_{2 k}\right\}
\end{aligned}
$$

as well as the adaptive law $\dot{\hat{\theta}}=\gamma \tau_{2}$ with

$\tau_{2} \stackrel{\text { def }}{=} \tau_{1}+w_{2} \sum_{k=1}^{N} z_{2 k}=W z$

where $c_{2}>0$ and $\gamma>0$ are design parameters and

$W=\left[\begin{array}{lllll}w_{1} & w_{2} & w_{2} & \cdots & w_{2}\end{array}\right]$

$z=\left[\begin{array}{lllll}z_{1} & z_{21} & z_{22} & \cdots & z_{2 N}\end{array}\right]^{\mathbf{T}}$

In fact, (21) is obtained by setting the first term between the brackets on the right side of (20) equal to $-c_{2} z_{2 k}$. The adaptive law $\dot{\hat{\theta}}=\gamma \tau_{2}$ is obtained by setting the second term between the brackets on the right side of (20) equal to zero. In (22) and (23), $\tau_{2}$ is referred to as the second tuning function, and $W$ and $z$ are called the regression and error vectors, respectively.

If $\zeta$ were null, an adaptive regulator implementing the control law (21) and the parameter adaptive law $\hat{\theta}=\gamma \tau_{2}$ would ensure that $\dot{V}=-c_{1} z_{1}^{2}-c_{2} \sum_{k=1}^{N} z_{2 k}^{2}$, which is actually a negative function of the state $z$. As $\zeta$ is not null, the above adaptive regulator is not sufficient to guarantee the intended control performances. In particular, the disturbing input $\zeta$ is likely to cause the parameter adaptive law $\dot{\hat{\theta}}=\gamma \tau_{2}$ to diverge, due to the integral nature of the law. To avoid such parameter drift, a modified version is used to constrain $\hat{\theta}$ to stay within a convex, bounded set that contains the true parameter $\theta$. One may consider this convex set to be the closed interval $C=\left[-M_{0}, M_{0}\right]$, where $M_{0}$ is any real number such that $M_{0} \geq|\theta|$. Then, the gradient algorithm with projection is proposed (see, e.g., Ioannou \& Sun, 1996):

$\dot{\hat{\theta}}=P\left(\gamma \tau_{2}\right) \stackrel{\text { def }}{=}\left\{\begin{array}{ll}\gamma \tau_{2} & \text { if } \hat{\theta}^{2}<M_{0}^{2} \\ 0 & \text { otherwise }\end{array}\right.$ or if $\left(\hat{\theta}^{2}=M_{0}^{2}\right.$ and $\left.\gamma \tau_{2} \hat{\theta} \leq 0\right)$

where $\hat{\theta}(0)$ is chosen such that $\hat{\theta}^{2}(0) \leq M_{0}^{2}$, and $P(\cdot)$ is a projection operator. It is readily seen that the above adaptive law keeps the estimate $\hat{\theta}$ within the convex bounded set $C$ at all times. That is, no risk of parameter drift exists anymore. More interestingly, it has been shown in the literature, e.g., (Ikhouane \& Krstic 1998; Ioannou \& Sun, 1996), that the projection operator $P(\cdot)$ exhibits the following key property:

$-\tilde{\theta} P\left(\gamma \tau_{2}\right) \leq-\tilde{\theta} \gamma \tau_{2}$

which simply means that the parameter convergence is not slower when using the modified adaptive law (25) than it was when using the standard law $\dot{\hat{\theta}}=\gamma \tau_{2}$. Hence, the projection feature in (25) not only prevents parameter drift, but also improves the convergence capability of the standard algorithm $\hat{\theta}=\gamma \tau_{2}$.

The adaptive regulator is thus defined by the control law (21) and the parameter adaptive law (25). Note that the assumption that $M_{0} \geq|\theta|$ is common in the robust adaptive control literature (e.g., Ioannou \& Sun, 1996). From a practical viewpoint, this is not an issue since the bound $M_{0}$ may be chosen to be arbitrarily large.

Remark 3.1. Note that the backstepping design technique has already been applied to control power converters, see, e.g., (Alvarez-Ramírez, Espinosa-Pérez, \& Noriega-Pineda, 2001; 
El Fadil \& Giri, 2007; Sira-Ramirez, García-Esteban, \& Zinober, 1996).

\section{Closed-loop performance analysis}

First, a state space representation of the closed-loop system is developed. Substituting the right side of (21) for $\mu_{k}$ in (17) yields:

$\dot{z}_{2 k}=-c_{2} z_{2 k}-z_{1}+w_{2} \tilde{\theta}+\frac{1}{N}\left(c_{1}-\frac{\hat{\theta}}{C_{e}}\right) \zeta \quad(k=1, \ldots, N)$

Eq. (27), together with (25), describe the closed-loop system. The latter is given the following, more compact, state space representation:

$\dot{z}=A_{z} z+W^{T} \tilde{\theta}+\varphi(\tilde{\theta}) \zeta$
$\dot{\tilde{\theta}}=-\dot{\hat{\theta}}=-P\left(\gamma \tau_{2}\right)$

where $A_{Z}$ is a skew-symmetric matrix defined by

$$
A_{z}=\left[\begin{array}{cccccc}
-c_{1} & 1 & 1 & 1 & \ldots & 1 \\
-1 & -c_{2} & 0 & 0 & \ldots & 0 \\
-1 & 0 & -c_{2} & 0 & \ldots & 0 \\
-1 & 0 & 0 & -c_{2} & \ldots & 0 \\
\vdots & \vdots & \vdots & \vdots & \vdots & \vdots \\
-1 & 0 & 0 & \ldots & 0 & -c_{2}
\end{array}\right]
$$

and $\varphi(\tilde{\theta})$ is a vector of dimension $(N+1)$, defined as follows:

$\varphi(\tilde{\theta})=\left[1, \frac{1}{N}\left(c_{1}-\frac{1}{R C_{e}}+\frac{\tilde{\theta}}{C_{e}}\right), \ldots, \frac{1}{N}\left(c_{1}-\frac{1}{R C_{e}}+\frac{\tilde{\theta}}{C_{e}}\right)\right]^{T}$

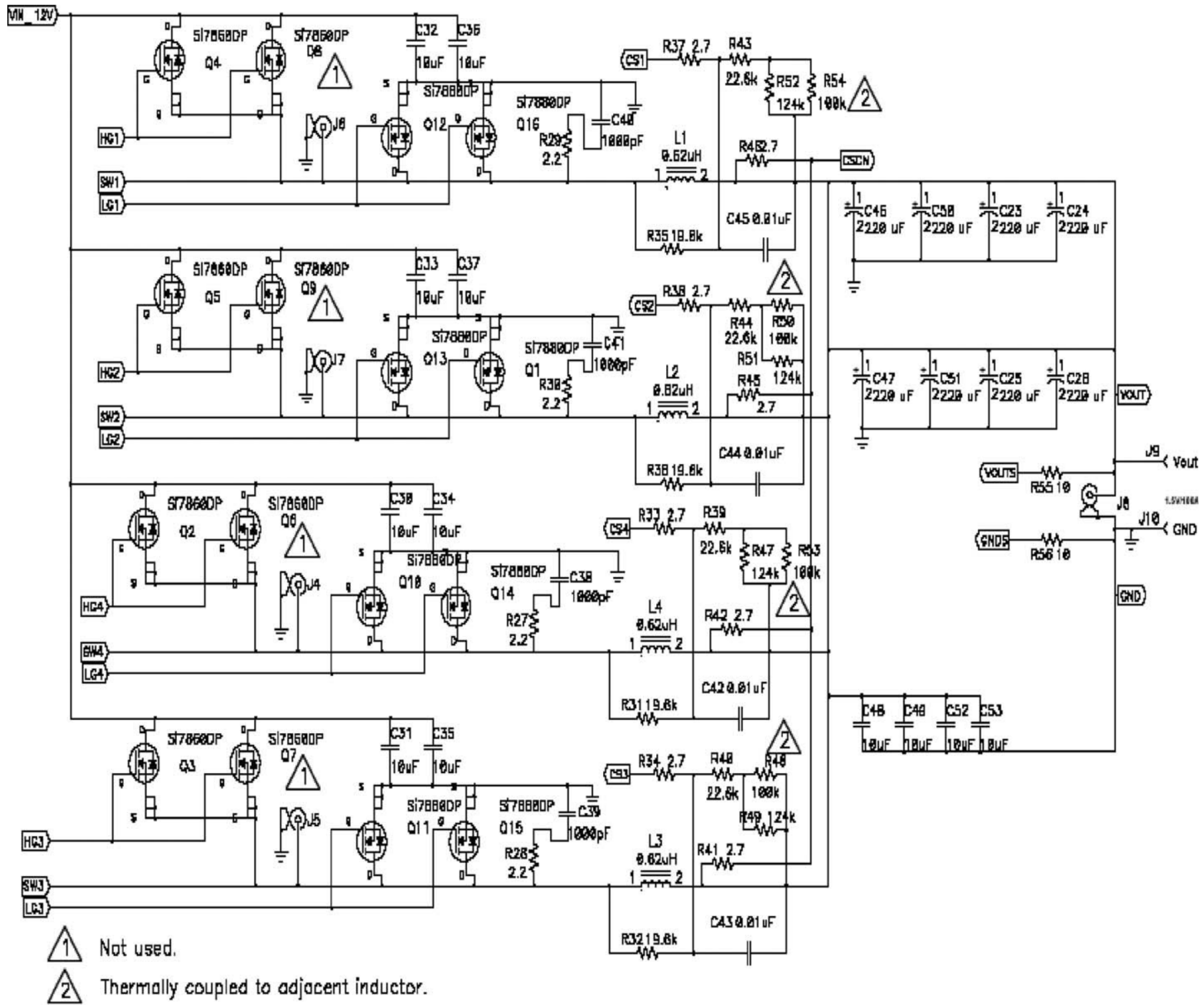

Fig. 4. Evaluation module TPS40090EVM-002. 


\subsection{Closed-loop analysis in the ideal case $\xi(t)=0$}

The stability of the closed-loop system described by (28) and (29) is now analyzed in the ideal situation, where $\xi(t)=0$. The main result is summarized in the following theorem:

Table 1

Parameters of the four-phase synchronous buck converters.

\begin{tabular}{lll}
\hline Parameter & Symbol & Value \\
\hline Number of phases & $N$ & 4 \\
Input voltage & $E$ & $12 \mathrm{~V}$ \\
Inductance value & $L$ & $0.62 \mu \mathrm{H}$ \\
Inductance ESR & $R_{L}=r_{L}$ & $1.75 \mathrm{~m} \Omega$ \\
Equivalent capacitor value & $C_{e}$ & $1800 \mu \mathrm{F}$ \\
Capacitor ESR & $R_{c}$ & $1.875 \mathrm{~m} \Omega$ \\
R-ON of switch S1 & $R_{1}$ & $4 \mathrm{~m} \Omega$ \\
R-ON of switch S2 & $R_{2}$ & $1.5 \mathrm{~m} \Omega$ \\
Switching frequency & $f_{s}$ & $420 \mathrm{kHz}$ \\
\hline
\end{tabular}

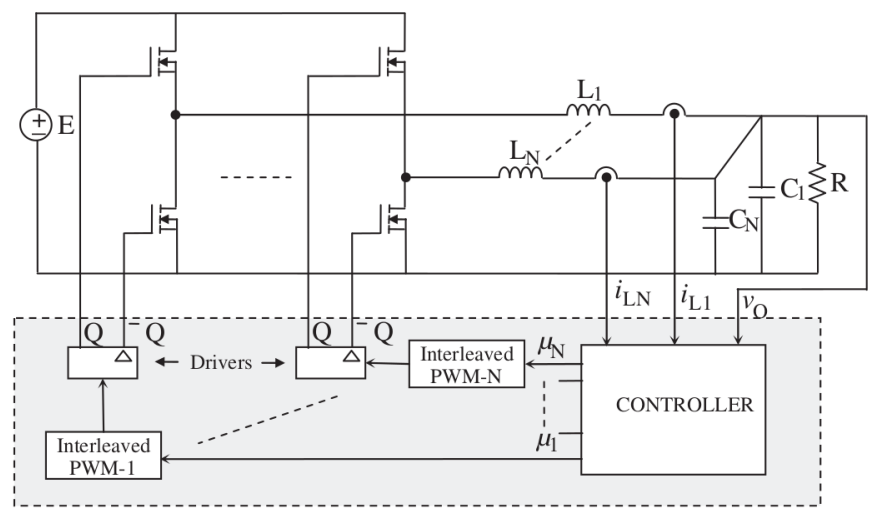

Fig. 5. Experimental control bench for interleaved buck power converters.

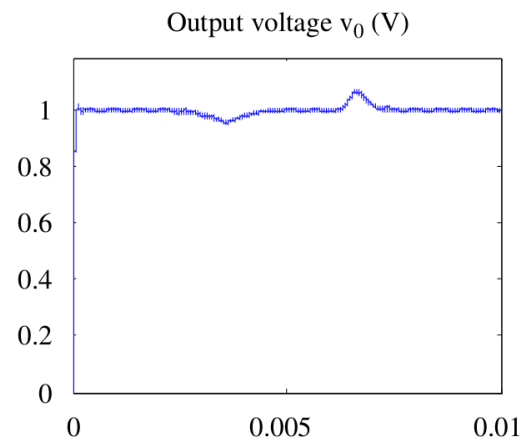

Load resistor $\mathrm{R}(\Omega)$

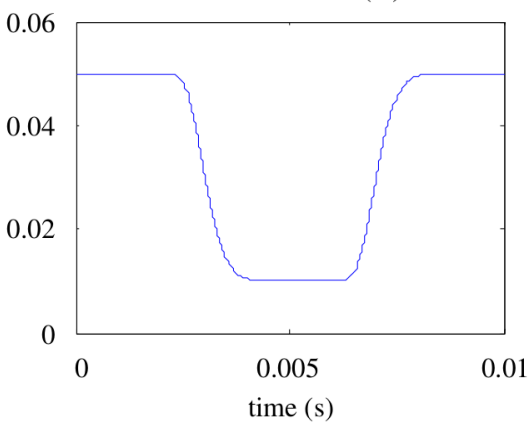

Theorem 1. Consider the closed-loop system consisting of the multiphase interleaved buck converter represented by model (2a) and (2b), where the load resistor $R$ is uncertain, and the adaptive controller composed of the control law (21) and the parameter update law (25). Then, the closed-loop system can be described by the state space representation (28) and (29), involving the error variables $z_{1}=\bar{v}_{0}-V_{d}$, $z_{2 k}=\left(\bar{i}_{L k} / C_{e}\right)-\left(\alpha_{1} / N\right)(k=1, \ldots, N)$, and $\tilde{\theta}=\hat{\theta}-\theta$. Furthermore, the system is globally asymptotically stable, implying that all control objectives have been achieved. Specifically, for any initial conditions:

(i) the output voltage $\bar{v}_{0}$ converges to its reference value $V_{d}$,

(ii) all currents $\bar{i}_{L k}$ converge to the same value $\left(C_{e} \alpha_{1}\right) / N$, ensuring perfect current sharing, and

(iii) the parameter estimate $\hat{\theta}$ converges to its true value $\theta$ whenever $\hat{\theta}(0) \in\left[-M_{0}, M_{0}\right]$.

\section{Proof. See Appendix A.}

\subsection{Closed-loop analysis in the general case $\xi(t) \neq 0$}

In this analysis, the disturbing term $\xi(t)$ is no longer neglected in the closed-loop system (28) and (29). The following theorem describes the obtained closed-loop performances.

Theorem 2. Consider the closed-loop system described in Theorem 1. There is a positive real $r^{*}$ such that, for all $R_{C} \in\left[0 r^{*}\right]$, there exists a real $v^{*}\left(R_{C}\right)>0$ such that, if $V(0)<v^{*}\left(R_{C}\right)$, then the Lyapunov function $V(t)$ converges to zero, and consequently:

(i) the output voltage $\bar{v}_{0}$ converges to its reference value $V_{d}$,

(ii) all currents $\bar{i}_{L k}$ converge to the same value $\left(C_{e} \alpha_{1}\right) / N$, ensuring perfect current sharing, and

(iii) the parameter estimate $\hat{\theta}$ converges to its true value $\theta$ whenever $\hat{\theta}(0) \in\left[-M_{0}, M_{0}\right]$.

Furthermore, if $R_{C} \rightarrow 0$, then $v^{*}\left(R_{C}\right) \rightarrow \infty$.

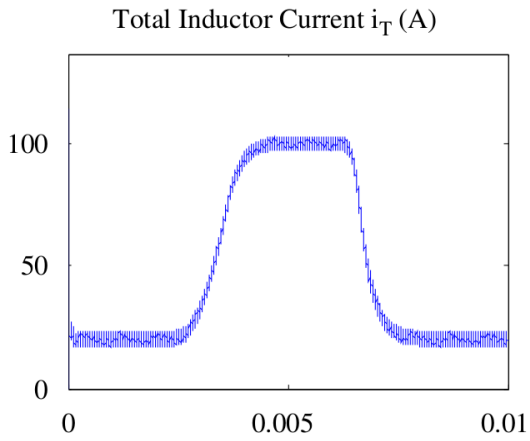

Duty ratio $\mu_{1}$

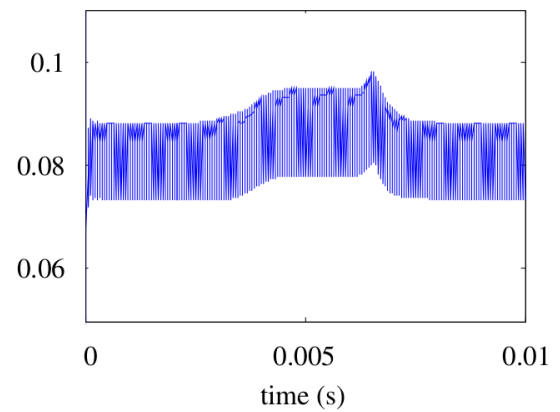

Fig. 6. Controller behavior in response to a step reference $V_{d}=1 \mathrm{~V}$ and changes in the load resistance. 
Proof. See Appendix B.

Remark 4.1. Theorem 2 shows that the error system (28) and (29) is asymptotically stable, and that its attraction region depends on the size of the parasitic resistance $R_{C}$. The size of the attraction region $\left(0 v^{*}\left(R_{C}\right)\right)$ increases as $R_{C}$ decreases. In particular, the attraction region becomes the whole positive real set $(0 \infty)$ in the ideal situation of $R_{C}=0$. The global asymptotic stability result of Theorem 1 is thus recovered.

\section{Simulation results}

The performances of the proposed adaptive controller are illustrated through numerical simulations. The controlled system
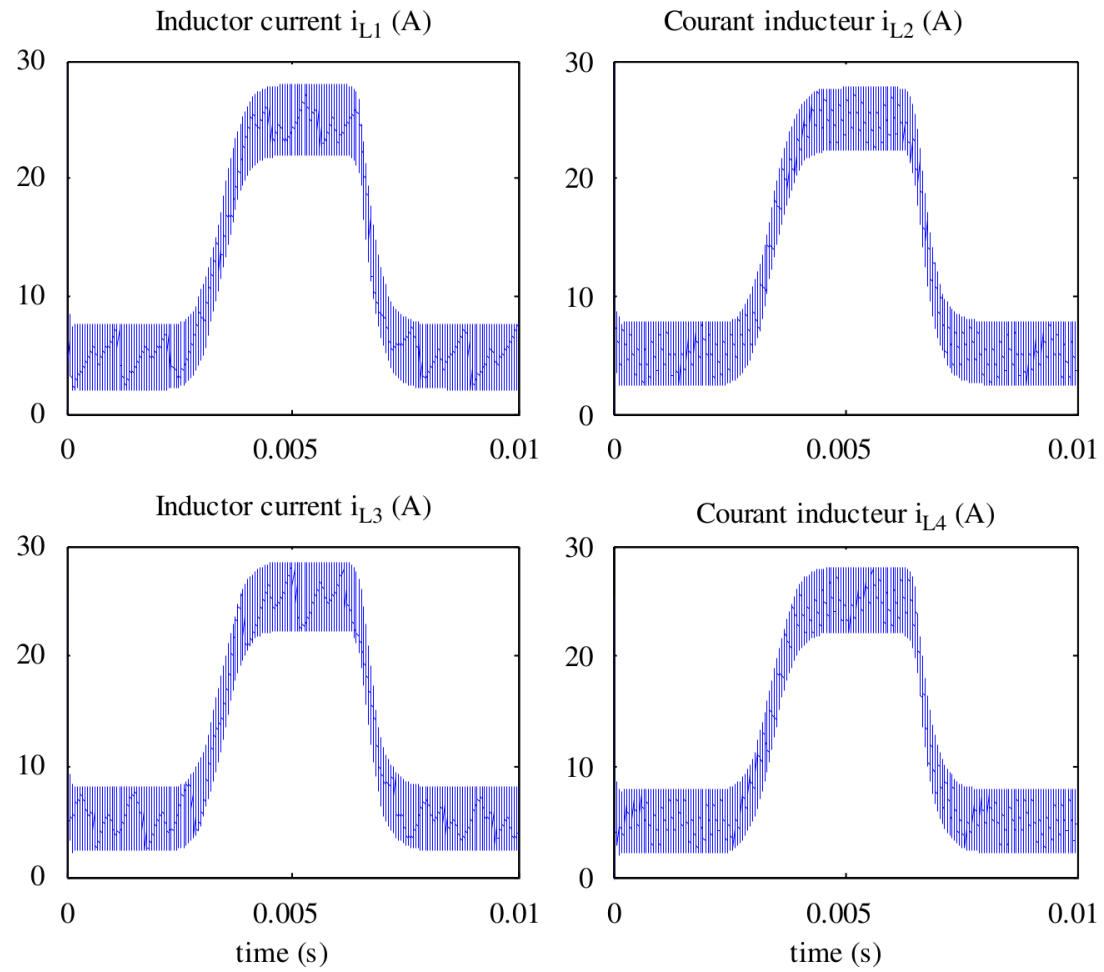

Fig. 7. Inductor currents in response to a step reference $V_{d}=1 \mathrm{~V}$ and changes in the load resistance.

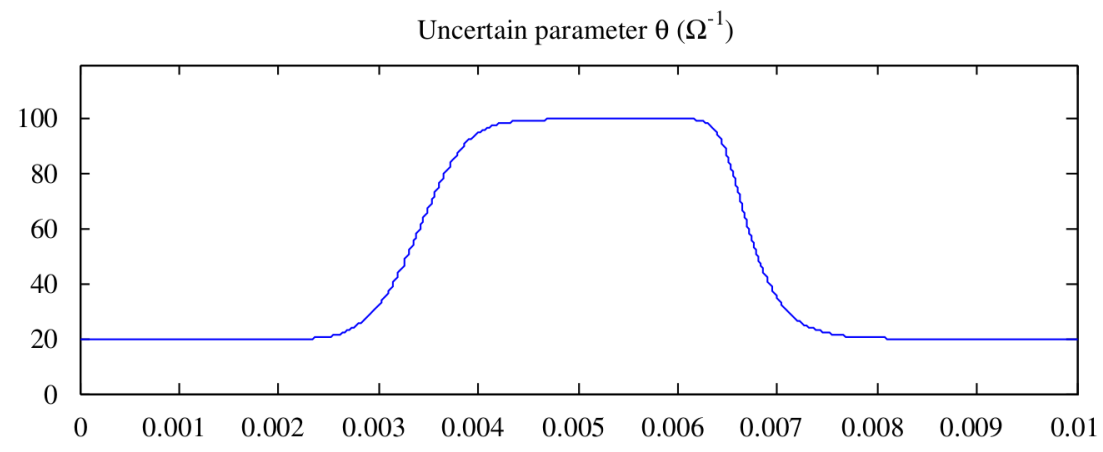

Estimate of $\theta\left(\Omega^{-1}\right)$

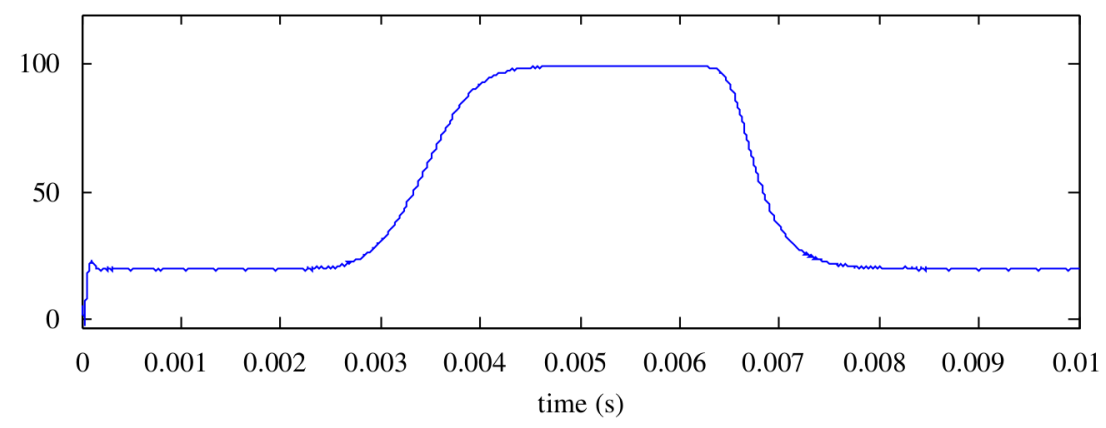

Fig. 8. Uncertain parameter and its estimate; if plotted in the same frame, the two curves cannot be distinguished after the time $t=5 \times 10^{-4}$ (s). 


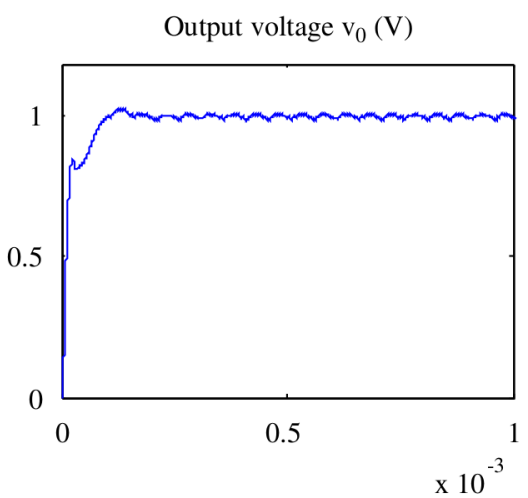

Estimate of $\theta\left(\Omega^{-1}\right)$

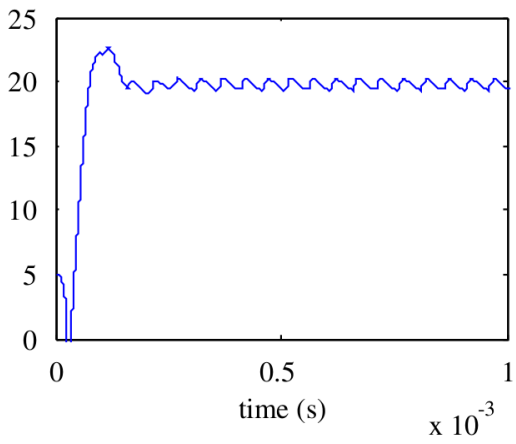

Total Inductor Current $\mathrm{i}_{\mathrm{T}}(\mathrm{A})$

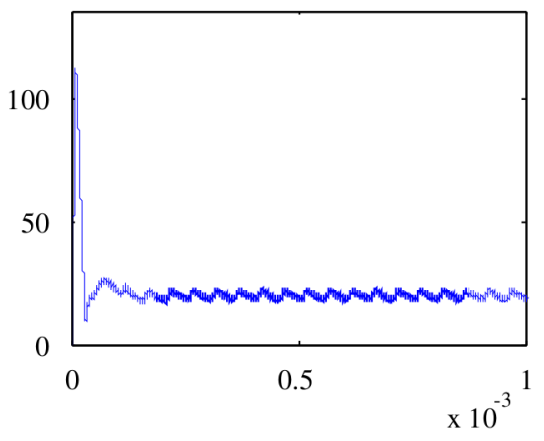

Duty ratio $\mu_{1}$

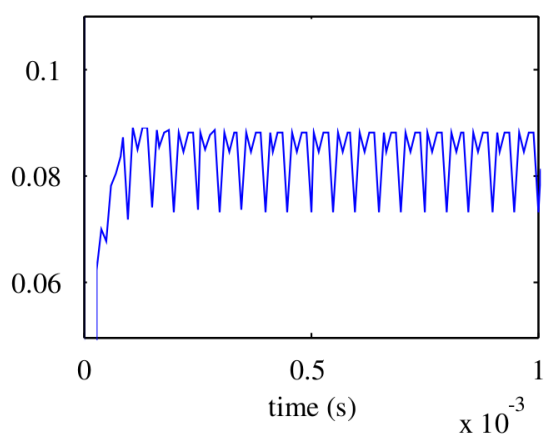

Fig. 9. Controller behavior during startup.

is the four-phase synchronous buck converter of Fig. 4; its characteristics are listed in Table 1 . These correspond to the Texas Instruments Evaluation Module TPS40090EVM-002 (see the user's guide, available at http://focus.ti.com/lit/ug/sluu195/ sluu195.pdf). The converter is numerically simulated using the real model (2a)-(2c), and all parasitic terms, especially the disturbance $\zeta(t)$, are included.

The experimental control bench is described by Fig. 5, and is simulated using the MATLAB software. The controller design parameters are given the following values: $c_{1}=11 \times 10^{4}, c_{2}=$ $8 \times 10^{4}, \gamma=4 \times 10^{-6}$, and $M_{0}=200$.

The resulting control performances are illustrated by Figs. 6-9. Fig. 6 illustrates the closed-loop system responses to a step reference $V_{d}=1 \mathrm{~V}$ (at time $t=0$ ), and successive load resistance jumps. The jumps vary between 0.01 and $0.05 \Omega$, yielding variations in the output current between 20 and $100 \mathrm{~A}$. It is seen that the control performances are quite satisfactory, despite uncertainty and variation in the load resistance. Figs. 7 and 8 show satisfactory current sharing between interleaved inductor currents, and perfect parameter estimation despite model uncertainty and variation. The zooms in Fig. 9 provide a detailed view of the controller behavior during startup.

\section{Conclusion}

The problem of controlling multiphase synchronous buck converters has been considered. A regulator, based upon the nonlinear average model (2), has been designed by applying a robust, adaptive version of the backstepping approach. When compared to previous works, the proposed regulator design presents some interesting new features: (i) the parasitic parameters $\left(R_{1}, R_{2}, R_{L}\right.$, and $\left.R_{C}\right)$ are considered in the converter model; in fact, the nonlinear feature of the model comes from the resistances $\left(R_{1}, R_{2}\right)$,

(ii) the load $R$ is allowed to be uncertain and time-varying, and

(iii) the non-standard adaptive law (21), which includes parameter projection, is used to cope, not only with uncertainty and variations in the load, but also with the effects of parasitic elements, which are assimilated into the modeling error $\zeta(t)$ in $(2 \mathrm{~b})$.

Using both formal analysis and simulation, it has been proven that the obtained adaptive regulator achieves the performances for which it was designed, namely:

(i) perfect asymptotic output voltage regulation,

(ii) excellent current sharing among modules, and

(iii) robustness to uncertainty in the load and parasitic parameters.

This is the first time that such a high level of performance has been formally achieved.

\section{Acknowledgment}

The authors are grateful to Professor F. Ikhouane from Universitat Politècnica de Catalunya of Barcelona, Spain for fruitful discussions during the preparation of this manuscript.

\section{Appendix A. Proof of Theorem 1}

Substituting the right side of (21) for $\mu_{k}$ in (20), and neglecting the term $\xi(t)$, the following derivative of the Lyapunov function $V$ 
is obtained:

$$
\begin{aligned}
\dot{V} & =-c_{1} z_{1}^{2}-c_{2} \sum_{k=1}^{N} z_{2 k}^{2}+\tilde{\theta}\left(\tau_{2}-\frac{\dot{\hat{\theta}}}{\gamma}\right) \quad(\text { using (22)) } \\
& =-c_{1} z_{1}^{2}-c_{2} \sum_{k=1}^{N} z_{2 k}^{2}+\frac{1}{\gamma}\left(\tilde{\theta} \gamma \tau_{2}-\tilde{\theta} P\left(\gamma \tau_{2}\right)\right) \quad(\operatorname{using}(25)) \\
& \leq-c_{1} z_{1}^{2}-c_{2} \sum_{k=1}^{N} z_{2 k}^{2} \quad(\operatorname{using}(26))
\end{aligned}
$$

In light of (19), inequality (A.1) shows that $\dot{V}$ is a negative semidefinite function of the state vector $\left[\begin{array}{ll}z^{T} & \tilde{\theta}\end{array}\right]$. Therefore, $\left[\begin{array}{ll}z^{T} & \tilde{\theta}\end{array}\right]=\left[\begin{array}{ll}0 & 0\end{array}\right]$ is globally stable. Applying LaSalle's Invariance Theorem, it further follows that the state vector $\left[z^{T} \tilde{\theta}\right]$ converges to the largest invariant set of (28) and (29) contained in the set $\left.\left\{\begin{array}{ll}z^{T} & \tilde{\theta}\end{array}\right] \in I R^{N+2} / \dot{V}=0\right\}$. In light of (A.1), the invariant set, denoted $M$, is itself contained in $E \stackrel{\text { def }}{=}\left\{\left[\begin{array}{ll}z^{T} & \tilde{\theta}\end{array}\right] I R^{N+2} / z=0\right\}$. Consequently, one has $z(t) \rightarrow 0$ as $t \rightarrow \infty$.

Now, one can prove by contradiction that the invariant set is limited to the origin, i.e., $M=\left\{\left[\begin{array}{ll}0 & 0\end{array}\right]\right.$. To this end, suppose that

$\left[\begin{array}{ll}0 & \lambda\end{array}\right] \in M$, for some $\lambda \neq 0$

Let $\left[z^{T}(0) \tilde{\theta}(0)\right]=\left[\begin{array}{ll}0 & \lambda\end{array}\right]$. It follows from the invariance of $M$ that:

$z(t)=0$ (and thus), $\dot{z}(t)=0$ ), for all $t>0$

which, together with (28), yields

$W(t) \tilde{\theta}(t)=0, \quad$ for all $t>0$

On the other hand, using (A.3) and (22), it can be shown that $\tau_{2}(t)=0$ for all $t>0$. This, in turn, yields:

$\dot{\tilde{\theta}}(t)=0 \quad$ (for all $t>0) \quad$ (using (25) and (29))

Since $\tilde{\theta}(0)=\lambda$, it follows from (A.5) that:

$\tilde{\theta}(t)=\lambda \neq 0 \quad($ for all $t \geq 0)$

Combining (A.4) and (A.6), it is shown that

$W(t)=0, \quad$ for all $t>0$

The following will show that (A.7) cannot hold. From (10), one has $w_{1}=-\bar{v}_{0} / C_{e}$, and from (6), $z_{1}=\bar{v}_{0}-V_{d}$. Since (A.3) ensures that $z_{1}=0$ (for all $t>0$ ), it follows that $w_{1}=V_{d} / C_{e} \neq 0$ for all $t>0$. This means that $W(t) \neq 0$ for all $t>0$, which contradicts (A.7). Hence, assumption (A.2) is false. Thus, $M=\left\{\left[\begin{array}{ll}0 & 0\end{array}\right]\right\}$ has been proven. This implies that $\hat{\theta}$ converges to its true value $\theta$. The proof of Theorem 1 is complete.

\section{Appendix B. Proof of Theorem 2}

Substituting the right side of (21) for $\mu_{k}$ in (20), the following derivative of the Lyapunov function $V$ is obtained:

$$
\begin{aligned}
\dot{V}=- & c_{1} z_{1}^{2}-c_{2} \sum_{k=1}^{N} z_{2 k}^{2}+\frac{1}{\gamma}\left(\tilde{\theta} \gamma \tau_{2}-\tilde{\theta} P\left(\gamma \tau_{2}\right)\right)+g(z, \tilde{\theta}) \zeta \\
& \quad(\text { using (22) and (25)) } \\
\leq & -c_{1} z_{1}^{2}-c_{2} \sum_{k=1}^{N} z_{2 k}^{2}+g(z, \tilde{\theta}) \zeta \quad \text { (using (26)) }
\end{aligned}
$$

with

$g(z, \tilde{\theta})=z_{1}+\frac{1}{N}\left(c_{1}-\frac{1}{R C_{e}}\right) \sum_{k=1}^{N} z_{2 k}+\frac{1}{N C_{e}} \tilde{\theta} \sum_{k=1}^{N} z_{2 k}$

Using (19), it follows from (B.1) that:

$\dot{V} \leq-\alpha_{0} V+\chi(z, \tilde{\theta}, \zeta)$ with

$\alpha_{0}=\min \left(c_{1}, c_{2}\right)$

$\chi(z, \tilde{\theta}, \zeta)=g(z, \tilde{\theta}) \zeta$

The next step is to show that the term $\chi(z, \tilde{\theta}, \zeta)$ in (B.3) can be bounded above by a polynomial function of $V$. It follows from (21) that:

$$
\begin{aligned}
E \mu_{k} & -\left(R_{1}-R_{2}\right) \bar{i}_{L k} \mu_{k} \\
= & L C_{e}\left\{\frac{1}{L C_{e}}\left(R_{L}+R_{2}\right) \bar{i}_{L k}+\left(\frac{1}{L C_{e}}-\frac{\hat{\theta}^{2}}{N C_{e}^{2}}\right) \bar{v}_{0}\right. \\
& \left.+\frac{\hat{\theta}}{N C_{e}^{2}} \bar{i}_{T}-\frac{w_{1}}{N} \dot{\hat{\theta}}+\left(\frac{c_{1}^{2}}{N}-1\right) z_{1}-\frac{C_{1}}{N} \sum_{k=1}^{N} z_{2 k}-C_{2} z_{2 k}\right\}
\end{aligned}
$$

which yields

$$
\begin{aligned}
E \sum_{k=1}^{N} \mu_{k}-\left(R_{1}-R_{2}\right) \sum_{k=1}^{N} \bar{i}_{L k} \mu_{k} \\
=L C_{e}\left\{\frac{1}{L C_{e}}\left(R_{L}+R_{2}\right) \bar{i}_{T}+\left(\frac{N}{L C_{e}}-\frac{\hat{\theta}^{2}}{C_{e}^{2}}\right) \bar{v}_{0}\right. \\
\left.\quad+\frac{\hat{\theta}}{C_{e}^{2}} \bar{i}_{T}-w_{1} \dot{\hat{\theta}}+\left(c_{1}^{2}-N\right) z_{1}-c_{1} \sum_{k=1}^{N} z_{2 k}-c_{2} \sum_{k=1}^{N} z_{2 k}\right\}
\end{aligned}
$$

This, together with $(2 \mathrm{c})$, implies that

$\zeta=R_{C} \eta(z, \tilde{\theta})$

with $\eta(z, \tilde{\theta})$ of the form

$$
\begin{aligned}
\eta(z, \tilde{\theta})= & \lambda_{1} z_{1}+\lambda_{2} \sum_{k=1}^{N} z_{2 k}+\lambda_{3} \tilde{\theta}+\lambda_{4} z_{1} \tilde{\theta} \\
& +\lambda_{5} \sum_{k=1}^{N} z_{2 k} \tilde{\theta}+\lambda_{6}\left(z_{1}+V_{d}\right)\left|P\left(\gamma \tau_{2}\right)\right|
\end{aligned}
$$

where the $\lambda_{i}=\lambda_{i}\left(R_{C}\right)(i=1, \ldots, 6)$ are real functions of the parasitic resistance $R_{C}$. For the sake of presentation, the expressions of these functions are precisely defined in Appendix C. These expressions show that

$\max _{0 \leq R_{C}<\infty}\left|\lambda_{i}\left(R_{C}\right)\right| \leq \lambda_{\max }<\infty \quad(i=1, \ldots, 7)$

where $\lambda_{\max }$ is a real constant, independent of $R_{C}$. On the other hand, from (25)

$\left|P\left(\gamma \tau_{2}\right)\right| \leq\left|\gamma \tau_{2}\right|$

which implies, due to (23), (24), (10), and (18), that

$\left|P\left(\gamma \tau_{2}\right)\right| \leq \frac{\gamma}{C_{e}}\left|z_{1}+V_{d}\right|\left(\left|z_{1}\right|+\left|\frac{c_{1}}{N}-\frac{1}{N R C_{e}}-\frac{\tilde{\theta}}{N C_{e}}\right|\left|\sum_{k=1}^{N} z_{2 k}\right|\right)$

In light of (B.10), (B.6), (B.7), (B.8), (B.5), and (B.2), it is easily seen that $\chi(t)$ is bounded above by a function of $V$ of the form

$|\chi(z, \tilde{\theta}, \zeta)| \leq R_{C}\left(\beta_{0} V+\beta_{1} V^{3}\right)$

for some constants $\beta_{0}>0, \beta_{1}>0$, independent of $R_{C}$. Combining (B.11) and (B.3), one obtains

$\dot{V} \leq-\alpha_{0} V+R_{C}\left(\beta_{0} V+\beta_{1} V^{3}\right)$

The rest of the analysis will show that $V$ remains bounded if $V(0)$ is within a certain neighborhood of the origin. To this end, the notation $r^{*} \stackrel{\text { def }}{=} \alpha_{0} / \beta_{0}$ and the following polynomial functions are introduced:

$f_{1}(v)=\left(\alpha_{0}-R_{C} \beta_{0}\right) v$ and $f_{2}(v)=R_{C} \beta_{1} v^{3}$ 


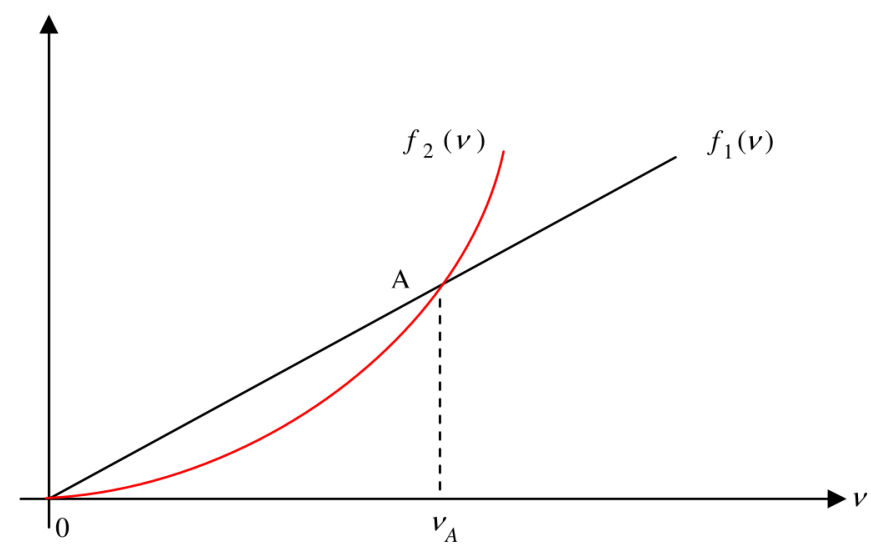

Fig. 10. Plot of functions $f_{1}(v)$ and $f_{2}(v)$.

It follows from (B.13) that if $R_{C}<r^{*}$, then $\alpha_{0}-R_{C} \beta_{0}>0$, and the plots of the above functions are as shown in Fig. 10. The intersection point, denoted $A$, has the abscissa

$v_{A}=\left(\frac{\alpha_{0}-R_{C} \beta_{0}}{R_{C} \beta_{0}}\right)^{1 / 2}$

As $f_{2}(v)-f_{1}(v)<0$ in the open interval $\left(0 v_{A}\right)$, it follows from (B.12) that $\dot{V}<0$ whenever $V \in\left(\begin{array}{ll}0 & v_{A}\end{array}\right)$. Consequently, if $V(0)<v_{A}$, then $V(t)$ converges to zero. Furthermore, it is readily seen from (B.14) that

$\lim _{R_{C} \rightarrow 0} v_{A}=\infty$

This establishes Theorem 2, with $v^{*}\left(R_{C}\right)=v_{A}$.

\section{Appendix C}

The constants $\lambda_{i}(i=1, \ldots, 7)$ in (B.7) are defined by the expressions:

$\lambda_{1}=a_{1}\left(\frac{1}{R}-c_{1} C_{e}\right)+a_{2}+b_{3} L C_{e}\left(c_{1}^{2}-N\right)$

$\lambda_{2}=a_{1} C_{e}-b_{3} L C_{e}\left(c_{1}+c_{2}\right)$

$\lambda_{3}=\left(a_{4}-\frac{a_{3}}{R}-a_{1}\right) V_{d}$

$\lambda_{4}=a_{4}-a_{3}\left(\frac{1}{R}-c_{1} C_{e}\right)-a_{1}$

$\lambda_{5}=-a_{3} C_{e}$

$\lambda_{6}=b_{3} L$

where

$a_{1}=b_{1}+b_{3}\left(R_{L}+R_{2}+\frac{L}{R C_{e}}\right)$

$a_{2}=b_{2}+b_{3}\left(N-\frac{L}{R^{2} C_{e}}\right)$ $a_{3}=\frac{b_{3} L}{C_{e}}$

$a_{4}=\frac{2 b_{3} L}{R C_{e}}$

$b_{1}=-\frac{1}{R+R_{c}}\left[\frac{R\left(R_{L}+R_{2}\right)}{L}+\frac{1}{C_{e}}\right]$

$b_{2}=-\frac{1}{R+R_{c}}\left[\frac{N R}{L}-\frac{1}{R C_{e}}\right]$

$b_{3}=\frac{R}{\left(R+R_{c}\right) L}$

\section{References}

Abu-Qahouq, J., Mao, H., \& Batarseh, I. (2004). Multiphase voltage-mode hysteretic controlled dc-dc converter with novel current sharing. IEEE Trans. Power Electron., 19(6), 1397-1407.

Alvarez-Ramírez, J., Espinosa-Pérez, G., \& Noriega-Pineda, D. (2001). Current-mode control of dc-dc power converters : A backstepping approach. In Proceedings of the 2001 IEEE international conference on control applications (pp. 190-195).

Berbel, N., Guerrero, J. M., Cruz, J., Miret, J., \& Castilla, M. (2005). One-cycle control for the parallel operation of synchronous buck converters. In Proceedings of the IEEE ISIE 2005 conference (pp. 795-798).

Chang, C., \& Knights, M. K. (1995). Interleaving technique in distributed power conversion systems. IEEE Trans. Circuits Syst. I, Fundam. Theor. Appl., 42(5), 245-251.

El Fadil, H., \& Giri, F. (2007). Backstepping based control of PWM DC-DC boost power converters. In Proceedings of the IEEE international symposium on industrial electronics (ISIE'07) (pp. 395-400), Vigo, Spain, June 4-7, 2007.

Huang, W., Schuellein, G., \& Clavette, D (2003). A scalable multiphase buck converter with average current share bus. In Proceedings of the IEEE APEC'03 (pp. 438-443).

Iannelli, L., Johansson, K. H., Jönsson, U. T., \& Vasca, F. (2008). Subtleties in the averaging of a class of hybrid systems with applications to power converters. Control Eng. Pract., 16(8), 961-975.

Ikhouane, F., \& Krstic, M. (1998). Adaptive backstepping with parameter projection: Robustness and asymptotic performance. Automatica, 34(4), 429-435.

Ioannou, P. A., \& Sun, J. (1996). Robust adaptive control. NJ: Prentice-Hall.

Krein, P. T., Bentsman, J., Bass, R., \& Lesieutre, B. (1990). On the use of averaging for the analysis of power electronic systems. IEEE Trans. Power Electron., 5, $182-190$.

Krstić, M., Kanellakopoulos, I., \& Kokotović, P. V. (1995). Nonlinear and adaptive control design. NY: Wiley.

Panov, Y., \& Jovanovic, M. M. (2001). Design consideration for 12-V/1.5-V, 50-A voltage regulator modules. IEEE Trans. Power Electron., 16(6), 776-783.

Perreault, D. J., \& Kassakian, J. G. (1997). Distributed interleaving of paralleled power converters. IEEE Trans. Circuits Syst. I Fundam. Theor. Appl., 44(8), $728-734$.

Saito, T., Tasaki, S., \& Torikai, H. (2005). Interleaved buck converters based on winner-take-all switching. IEEE Trans. Circuits Syst., 52(8), 1666-1672

Sira-Ramirez, H., García-Esteban, M., \& Zinober, A. S. I. (1996). Dynamical adaptive pulse-width modulation control of dc to dc power converters : A backstepping approach. Int. J. Control, September, 205-222.

Zhang, M. T., Jovanovic, M. M., \& Lee, F. C. (1996). Design consign considerations for low-voltage on-board DC/DC modules for next generations of data processing circuits. IEEE Trans. Power Electron., 11(2), 328-337.

Zhou, X., Xu, P., \& Lee, F. C. (2000). A novel current-sharing control technique for low-voltage high-current voltage regulator module applications. IEEE Trans. Power Electron., 15(6) 1153-1162. 